\title{
Influence of different formulations and process parameters during the preparation of drug-loaded PLGA microspheres evaluated by multivariate data analysis
}

\author{
JAKUB VYSLOUŽIL ${ }^{1}$ \\ PETR DOLEŽEL ${ }^{1}$ \\ MARTINA KEJDUŠOVÁ ${ }^{1}$ \\ ELIŠKA MAŠKOVÁ ${ }^{1}$ \\ JOSEF MAŠEK ${ }^{2}$ \\ ROBERT LUKÁČ ${ }^{2}$ \\ VRATISLAV KOŠŤÁL ${ }^{3}$ \\ DAVID VETCHÝ \\ KATEŘINA DVOŘÁČKOVÁ ${ }^{1, *}$ \\ ${ }^{1}$ Department of Pharmaceutics \\ Faculty of Pharmacy \\ University of Veterinary \\ and Pharmaceutical Sciences, Brno \\ 61242 Brno, Czech Republic \\ ${ }^{2}$ Department of Toxicology \\ Pharmacology and Immunotherapy \\ Veterinary Research Institute \\ 62100 Brno, Czech Republic \\ ${ }^{3}$ Tescan, 62300 Brno-Kohoutovice \\ Czech Republic
}

\begin{abstract}
The main objective of this study was to evaluate the influence of the formulation and process parameters on PLGA microparticles containing a practically insoluble model drug (ibuprofen) prepared by the o/w solvent evaporation method. Multivariate data analysis was used. The effects of altered stirring speed of a mechanical stirrer $(600,1000$ rpm), emulsifier concentrations (PVA concentration $0.1 \%$, $1 \%$ and solvent selection (dichloromethane, ethyl acetate) on microparticle characteristics (encapsulation efficiency, drug loading, burst effect) were observed. It was found that with increased stirring speed, the PVA concentration or the use of ethyl acetate had a significantly negative effect on encapsulation efficiency. In addition, ethyl acetate had an adverse effect on the burst effect, while increased stirring speed had the opposite effect. Drug load was not affected by any particular variable, but rather by the interactions of evaluated variables.
\end{abstract}

Keywords: solvent evaporation, encapsulation, PLGA, controlled release, burst effect

Accepted June 16, 2014

In the last decade, poly(lactic-co-glycolic acid) (PLGA) received great interest in the medical and pharmaceutical field because of its biodegradability, toxicological safety and desirable biocompatibility. It is one of the small number of synthetic polymers that has been approved by the FDA for human clinical use. Nowadays, PLGA is widely applied in controlled drug delivery systems, including nano- and micro-particles and implants (1). PLGA-based microparticles are well established as advanced drug delivery systems, allowing drug protection and controlled release upon parenteral administration (2). PLGA microspheres are considered to be an important carrier for the encapsulation of water-insoluble drug candidates in the early stages of drug development (3). They offer various advantages over other controlled drug delivery systems, including: (i) the possibility to

\footnotetext{
*Correspondence; e-mail: dvorackovak@vfu.cz
} 
accurately control the drug release kinetics over a period of days to months; (ii) complete biodegradability (avoiding the removal of empty remnants upon drug exhaust); (iii) good biocompatibility, even within brain tissue $(4,5)$. However, the design of this type of advanced drug delivery system is not straightforward, because many factors influence the resulting drug release rate from PLGA microparticles, including the method of preparation. One of the most common methods of preparing PLGA microparticles is the single emulsion solvent evaporation method, which consists of four major steps: (i) dissolution or dispersion of the bioactive compound in an organic solvent containing the matrix-forming polymer; (ii) emulsification of this organic phase in a second, continuous (frequently aqueous) phase immiscible with the first phase; (iii) extraction of the solvent from the dispersed phase by the continuous phase, which is optionally accompanied by solvent evaporation transforming the droplets into solid microspheres; (iv) harvesting and drying of microspheres (6). The first two steps primarily affect the microparticle size distribution while the last two steps determine the microparticle morphology and have an important influence on the microparticle encapsulation and release behavior (7).

The main objective of the experiment was to prepare drug-loaded PLGA microspheres containing practically insoluble ibuprofen (IBU), which served as the model drug. Several formulation and process parameters of the oil in water $(\mathrm{o} / \mathrm{w})$ single emulsion method were modified, such as alteration of the stirring speed during solvent evaporation, emulsifier concentration and the type of organic solvent to enhance ibuprofen encapsulation. The main section is devoted to the expression of the statistical significance of the above-mentioned formulation and process parameters on PLGA particle characteristics, such as shape and size, encapsulation efficacy and in vitro drug release. The full factorial design method and partial least squares PLS-2 regression were regarded the best methods for evaluating the impact of parameters that were otherwise difficult to compare.

\section{EXPERIMENTAL}

\section{Materials}

Ibuprofen (Zentiva, Czech Republic) was the model drug, PLGA Resomer ${ }^{\circledR}$ RG $504 \mathrm{~S}$ (Boehringer Ingelheim, Pharma GmbH \& Co, Germany) was used as the polymer carrier. Dichloromethane (DM, Penta, Czech Republic) and ethyl acetate (EA, Dr. Kulich Pharma, Czech Republic) were used as solvents and polyvinylalcohol (PVA) served as emulsifier. A phosphate buffer at $\mathrm{pH} 6.8$ (dodecahydrate sodium hydrogenphosphate, potassium dihydrogenphosphate, both Merck KGaA, Germany) was applied as the dissolution medium. All materials were of Ph. Eur. quality.

\section{Sample preparation}

Drug-loaded PLGA microparticles were prepared by the o/w solvent evaporation method. In order to form an o/w-type emulsion, $150 \mathrm{mg}$ of the drug and $700 \mathrm{mg}$ of the polymer were dissolved in $5 \mathrm{~mL}$ of either dichloromethane or ethyl acetate. The solution was homogenized for 1 minute using an Ultra-Turrax (T25 basic, IKA-Werke, Germany) at 20,000 rpm for one minute to dissolve the polymer completely and then it was emulsified all at once into $800 \mathrm{~mL}$ of the aqueous continuous phase containing either $0.1 \%(\mathrm{~m} / \mathrm{m})$ or 
J. Vysloužil et al.: Influence of different formulations and process parameters during the preparation of drug-loaded PLGA microspheres evaluated by multivariate data analysis, Acta Pharm. 64 (2014) 403-417.

$1 \%(\mathrm{~m} / \mathrm{m})$ PVA. The emulsion was stirred with a mechanical stirrer with a pitched-blade impeller (Heidolph RZR 2021, Sigma Aldrich, USA) at either 600 or $1000 \mathrm{rpm}$ for 2 hours to ensure complete solvent evaporation. The prepared microparticles were collected on a fine mesh sieve with $80 \mu \mathrm{m}$-sized openings, washed three times with purified water and dried at $25{ }^{\circ} \mathrm{C}$ in a cabinet drier (HORO-048B, Dr. Hofmann $\mathrm{GmbH}$, Germany) for $24 \mathrm{~h}$ before further testing. All samples were prepared in triplicate and marked according to the altered parameters: DM/ET stands for organic solvent (dichloromethane or ethyl acetate), L/H expresses low or high PVA concentration and 600/1000 determines the stirring speed per minute. Sample characteristics are shown in Table I.

Table I. Variables in the preparation of microparticle samples

\begin{tabular}{cccc}
\hline Sample & Organic solvent & PVA concentration (\%) & Stirring speed (rpm) \\
\hline DM-L-600 & DM & 0.1 & 600 \\
DM-L-1000 & DM & 0.1 & 1000 \\
EA-L-600 & EA & 0.1 & 600 \\
EA-L-1000 & EA & 0.1 & 1000 \\
DM-H-600 & DM & 1 & 600 \\
DM-H-1000 & DM & 1 & 1000 \\
EA-H-600 & EA & 1 & 600 \\
EA-H-1000 & EA & 1 & 1000 \\
\hline
\end{tabular}

\section{Particle distribution}

The size-based mean diameter and size distribution of the microparticles were measured with a laser diffraction particle size distribution analyzer (Horiba Partica LA-300, Horiba Ltd., Japan). $100 \mu \mathrm{L}$ of a prediluted microparticle sample $\left(0.5 \mathrm{~g} \mathrm{~mL}^{-1}\right.$ of phosphate buffered saline) was injected into a cuvette-type fraction cell (filled with $10 \mathrm{~mL}$ of degassed and filtered phosphate buffered saline, $\mathrm{pH}$ 7.2) provided with a magnetic stirrer to prevent non-homogenous distribution due to the sedimentation of particles. All samples were measured immediately after the application into the cuvette and were analyzed for number-weighted size distribution.

\section{Scanning electron microscopy}

The morphology and surface topography of the microparticles were examined by scanning electron microscopy. The samples were mounted directly onto the SEM sample holder using double-sided tape and then coated with a 10-nm thick layer of gold powder and images were taken using a scanning electron microscope (MIRA3, Tescan, Czech Republic) at accelerating voltage of $5.0 \mathrm{kV}$. 
J. Vysloužil et al.: Influence of different formulations and process parameters during the preparation of drug-loaded PLGA microspheres evaluated by multivariate data analysis, Acta Pharm. 64 (2014) 403-417.

\section{Drug content}

Ibuprofen content in the drug loaded microparticles was determined by dissolving an appropriate amount of dried PLGA-based microparticles ( 10 mg of ibuprofen) in $25 \mathrm{~mL}$ of an appropriate organic solvent (ethyl acetate or dichloromethane). The absorbance of samples was measured at $264 \mathrm{~nm}$ using a UV/Vis spectrometer (Lambda 25, Perkin Elmer, USA). Encapsulation efficiency (EE) and drug load (DL) were determined from the values obtained using the following equations $(8,9)$ :

$$
\text { Encapsulation efficiency }=\frac{c_{\mathrm{s}}}{c_{\mathrm{t}}} 100(\%)
$$

where $c_{\mathrm{s}}$ corresponds to the actual ibuprofen content and $c_{\mathrm{t}}$ corresponds to the theoretical drug load. The assay was carried out in triplicate and the results were expressed as mean values and standard deviations (SD).

$$
\text { Drug load }=\frac{m_{1}}{m_{2}} 100(\%)
$$

where $m_{1}$ is the drug mass in microparticles, $m_{2}$ is the gross mass of microparticles. The assay was carried out in triplicate. The results are expressed as mean values and SD.

The effectiveness of the process was also evaluated by the yield, calculated by the following equation (10):

$$
\text { Yield }=\frac{m_{2}}{m_{t}} 100(\%)
$$

where $m_{2}$ is the gross mass of microparticles, $m_{\mathrm{t}}$ is the total mass of the drug and polymer used for microparticle preparation.

\section{In vitro release studies}

The in vitro release studies of drug-loaded beads were carried out using the paddle method in an automatic dissolution apparatus (SOTAX AT 7 On-Line System, Donau Lab, Switzerland) at $100 \mathrm{rpm}$. The dissolution medium volume used was $500 \mathrm{~mL}$ of phosphate buffer (pH 6.8) kept at $37.0 \pm 0.5^{\circ} \mathrm{C}$. Samples were analyzed using a UV spectrophotometer (Lambda 25, Perkin Elmer, USA) at $264 \mathrm{~nm}$. The samples for the dissolution test were weighed with respect to their encapsulation efficiency, so that $50 \mathrm{mg}$ of ibuprofen was in each sample. The in vitro drug release was observed for 72 hours. The dissolution test was carried out with six samples of each batch and the results were expressed as average values and SD.

\section{Similarity factor analysis}

Similarity factor $f_{2}$ was originally designed for dissolution profiles comparing the originals and the generics (11). It can be also used for the drug release profile comparison of samples prepared with different parameters. Similarity factor values ranged between 0 and 100 . If $f_{2} \geq 50$, drug release profiles were over $90 \%$ similar. If $f_{2}<50$, release profiles were not similar and the observed influence of the formulation or process variables was considered to be significant. Standard deviations of the mean data did not exceed $9.1 \%$, so 
the conditions for the variation (20\% maximum for the earlier points, $10 \%$ maximum for the rest) were met. No more than one measurement was considered after $85 \%$ of dissolution (12).

$$
f_{2}=50 \times \log \left\{\left[1+\left(\frac{1}{n}\right) \sum_{i=1}^{n}\left|R_{\mathrm{i}}-T_{\mathrm{i}}\right|^{2}\right]^{-0,5} \times 100\right\}
$$

$R_{\mathrm{i}}$ - drug amount (\%) released at time interval $i$; reference sample

$T_{\mathrm{i}}$ - drug amount (\%) released at time interval $i$; tested sample

$n$ - total number of samplings

\section{Factorial design}

The experiment was planned as a $2^{2}$ full factorial design with three replicated measurements, i.e., 12 data points (Table I), to evaluate the influence of several variables (PVA concentration, stirring speed) and their interaction with response variables (encapsulation efficiency, drug loading, burst effect value) for each solvent separately. Individual experiments were performed in random order. The resulting data were analyzed by Unscrambler $\mathrm{X}$ (v. 1.3, Camo software) with the application of multiple linear regressions. For each response-variable, the results were expressed as the regression model in the form:

$$
Y=\beta_{0}+\beta_{1} X_{1}+\beta_{2} X_{2}+\beta_{12} X_{1} X_{2}+e
$$

where $Y$ is the response variable, $X_{1}$ to $X_{3}$ are technological variables, $\beta_{0}$ is a constant (intercept), $\beta_{1}$ to $\beta_{12}$ are regression coefficients representing an individual effect or interaction, $e$ is the residual, i.e., the difference between the observed and predicted values of Y. MLR models can be described on the basis of goodness-of-fit coefficients. R-square describes variability explained by the model, R-square of prediction expresses predictive ability of the model. C.V. (Coefficient of Variation) is the average modeling error expressed as percentage of the mean (13). Model significance was tested using ANOVA (Analysis of Variance); it was also used for evaluation of individual and interaction effects.

Analyzed data, including yield and particle size (Table I), were also evaluated by a principal component analysis (PCA) to study systematic variability and the relationships between variables and objects.

\section{RESULTS AND DISCUSSION}

\section{Particle size and morphological properties}

Effect of stirring speed. - The prepared microparticles with the mean size ranging between 90.7 to $382.8 \mu \mathrm{m}$ showed particle size dependence on the stirring speed during solvent evaporation. The results in Table II confirmed that the microparticle mean size decreased with an increase in the stirring speed, which is a very well-documented effect $(14,15,16)$. The force of higher stirring speed distributes the internal phase into smaller droplets, resulting in the formation of smaller microparticles (14). 
J. Vysloužil et al.: Influence of different formulations and process parameters during the preparation of drug-loaded PLGA microspheres evaluated by multivariate data analysis, Acta Pharm. 64 (2014) 403-417.

Table II. Characteristics of drug-loaded microparticles

\begin{tabular}{cccccc}
\hline Sample & DL $(\%)$ & EE $(\%)$ & Yield $(\%)$ & $\begin{array}{c}\text { Mean size } \\
(\mu \mathrm{m})\end{array}$ & $\begin{array}{c}\text { Initial drug release } \\
\text { after 30 min }(\%)\end{array}$ \\
\hline DM-L-600 & $18.2 \pm 0.1$ & $79.8 \pm 0.5$ & $77.3 \pm 5.1$ & 382.8 & $20.0 \pm 3.5$ \\
DM-L-1000 & $17.0 \pm 0.3$ & $57.4 \pm 0.3$ & $59.6 \pm 5.2$ & 254.9 & $23.6 \pm 3.4$ \\
EA-L-600 & $17.5 \pm 0.4$ & $58.2 \pm 1.6$ & $40.7 \pm 6.6$ & 174.6 & $9.0 \pm 3.9$ \\
EA-L-1000 & $16.2 \pm 0.4$ & $22.2 \pm 1.4$ & $24.2 \pm 4.6$ & 169.7 & $14.7 \pm 3.9$ \\
DM-H-600 & $13.9 \pm 0.5$ & $44.6 \pm 0.6$ & $56.5 \pm 5.0$ & 284.3 & $18.0 \pm 3.8$ \\
DM-H-1000 & $20.0 \pm 2.4$ & $23.2 \pm 2.5$ & $29.3 \pm 6.2$ & 173.5 & $29.1 \pm 9.7$ \\
EA-H-600 & $19.0 \pm 0.4$ & $40.4 \pm 0.4$ & $54.0 \pm 3.8$ & 90.7 & $9.0 \pm 3.5$ \\
EA-H-1000 & $17.5 \pm 0.4$ & $16.7 \pm 0.5$ & $16.8 \pm 7.1$ & 146.6 & $9.5 \pm 0.8$ \\
\hline
\end{tabular}

Effect of PVA concentration. - It is also evident from Table II that particle size was dependent on the external phase viscosity, as the increasing PVA concentration decreased particle size. The particle size of samples prepared with $0.1 \%$ PVA ranged between 169.7 and $382.8 \mu \mathrm{m}$, while the size of microparticles prepared with $1 \%$ PVA had values from 90.7
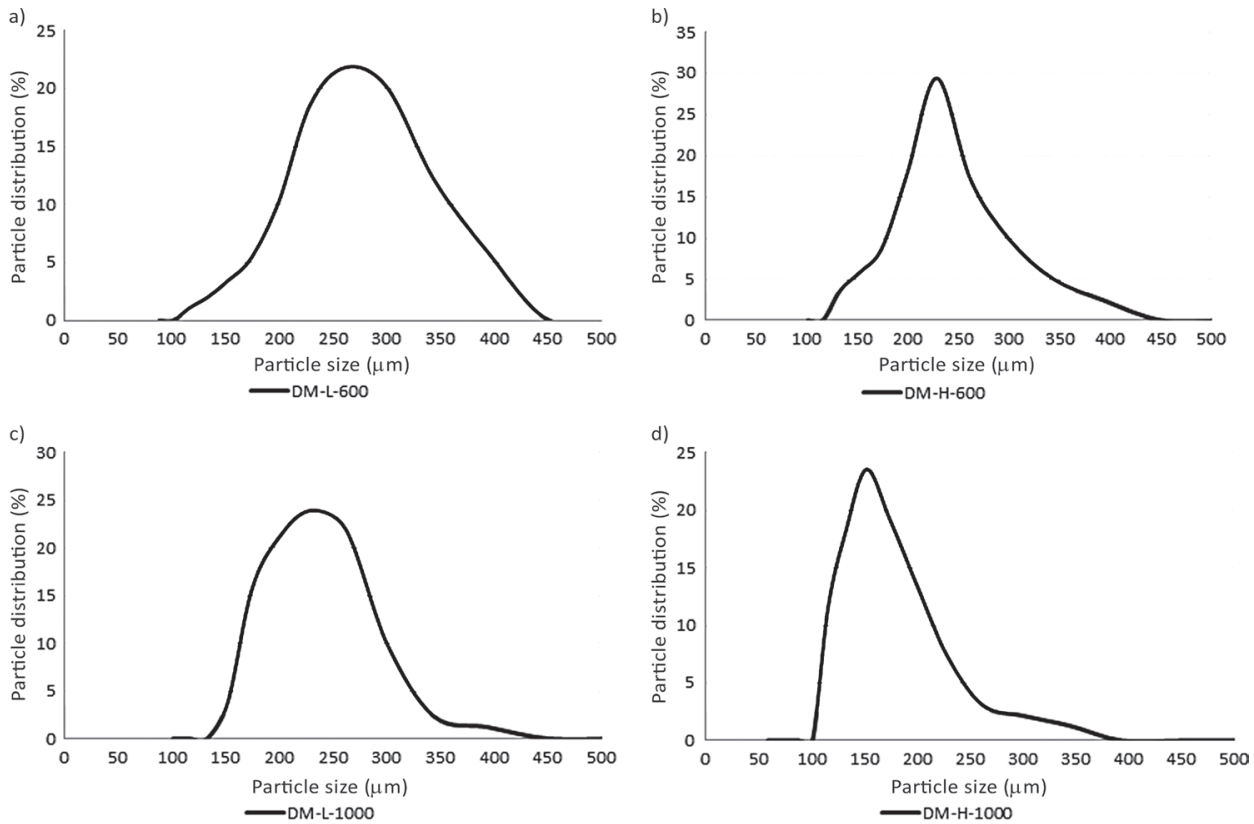

Fig. 1. Particle size distribution of PLGA (Resomer ${ }^{\circledR}$ RG 504S) microparticles prepared with dichloromethane as organic solvent: a) DM-L-600, b) DM-H-600, c) DM-L-1000, d) DM-H-1000. 
a)

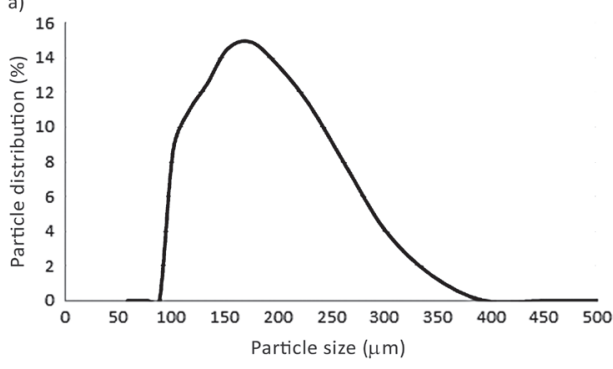

- EA-L-600

c)

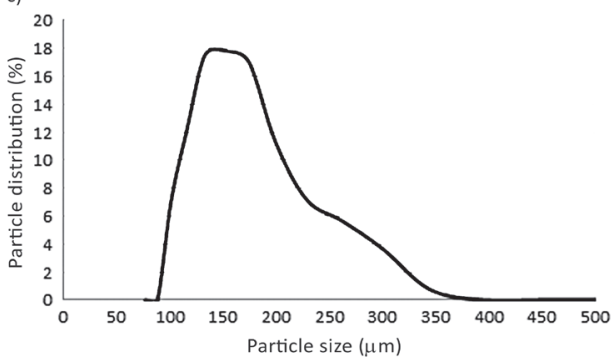

-EA-L-1000

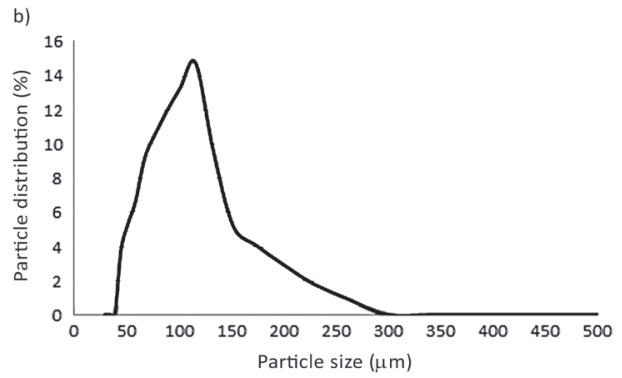

- EA-H-600

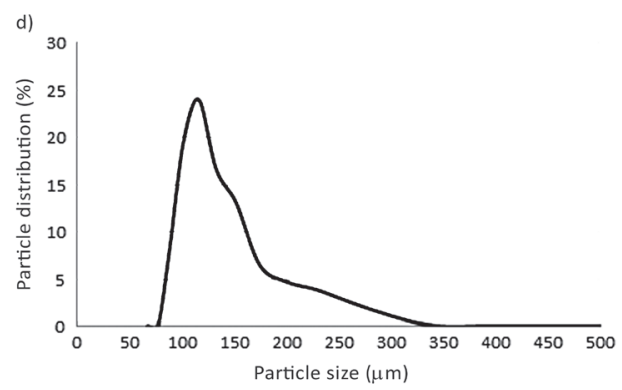

- EA-H-1000

Fig. 2. Particle size distribution of PLGA (Resomer ${ }^{\circledR}$ RG 504S) microparticles prepared with ethyl acetate as organic solvent: a) EA-L-600, b) EA-H-600, c) EA-L-1000, d) EA-H-1000.

to $284.3 \mu \mathrm{m}$. Increased PVA concentration ensured better system stabilization against coalescence of the emulsion and therefore led to formation of smaller microparticles (17). Better emulsion stability also resulted in narrower particle size distribution in all samples, as seen in Figs. 1 and 2. Another well-observed effect (18) was that the value of the PVA concentration influenced also the shape of prepared microparticles. Fig. 3 shows that DML-600 provided spherical microparticles with holes in the surface, while DM-H-600 gave compact, spherical, regular and more uniform microparticles.

Effect of the organic solvent choice. - As observed in Fig. 3 and Table II, microparticles prepared with dichloromethane provided regular, highly spherical microparticles with a smooth surface, characterized by larger particle size in contrast to the smaller, irregularlyshaped microparticles created when ethyl acetate was used. A typical feature of ethyl acetate microspheres is their rough surface. An explanation could be that the polarity of ethyl acetate is higher compared to dichloromethane, resulting in microparticles of lower quality (19).

Encapsulation process. - Drug loading of the prepared samples ranged between 13.9 and $20.0 \%$, encapsulation efficiency had values from 16.7 to $79.8 \%$ (Table II). For evaluation of the encapsulation process, i.e., influence of the parameters on encapsulation efficiency and drug loading, MLR models for both organic solvents were used separately, four models in all. Regression diagnostics of all models had very good values (R-square $>0.8, R-$ square predicted $>0.8$, C.V. $<3 \%$, models $p<0.001$ ). 


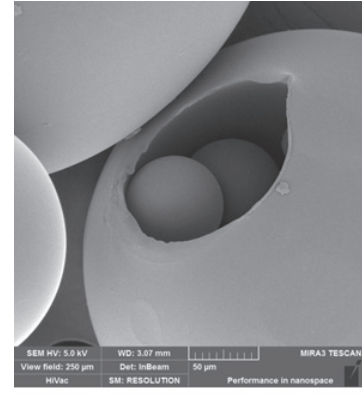

a

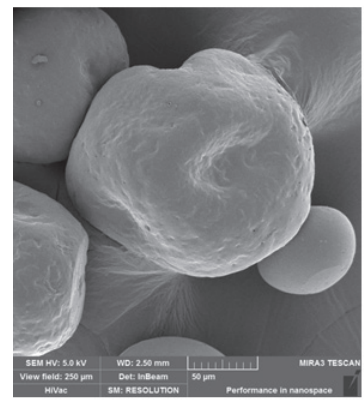

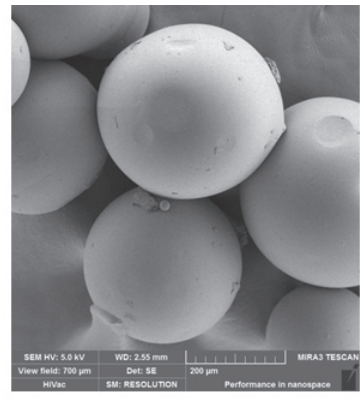

b

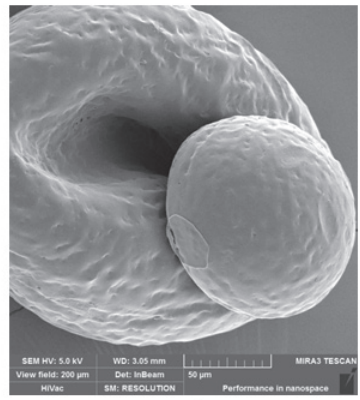

Fig. 3. SEM photographs of the surface of PLGA microparticles corresponding to: a) DM-L-600, b) DM-H-600, c) EA-L-600, d) EA-H-600.

Plots of interactions were used to compare the influence of variables on the effect. They represent an average value of factor-effect in dependence on the level of the second factor. In case of encapsulation efficiency for ethyl acetate samples (Fig. 4a), positive significance ( $p<$ 0.001) of the PVA concentration-stirring speed interaction was found. The plot shows that the influence of PVA concentration depends on the stirring speed. On the other hand, results for dichloromethane samples reveal a significant effect of individual variables $(p<0.001$ for both), as shown in Fig. 4b. Both connecting lines between the effect averages are parallel, i.e., for both PVA concentrations, the increase in stirring speed equals an approximately identical decrease in encapsulation efficiency. In case of both solvents, the maximum of encapsulation efficiency was reached with lower PVA concentration and slower stirring speed, which is in agreement with the literature. Increase in the stirring speed delivers greater energy to the system, resulting in an increased breakdown of the forming microparticles and lower encapsulation efficiency (20). It was also found that an excessive increase of PVA concentration can lead to lower encapsulation efficiency, probably due to higher viscosity $(21,22)$. Of both solvents used, ethyl acetate was found to provide lower values for encapsulation efficiency. Higher polarity of ethyl acetate, and therefore higher miscibility with the external phase, was probably responsible for the formation of a less stable o/w emulsion. In the EA/ PVA system, the drug is transferred to the external phase more frequently and a smaller number of microparticles is produced, resulting in lower encapsulation efficiency (23).

In case of drug loading in samples prepared with ethyl acetate (Fig. 5a), the significant effect of individual variables is obvious $(p<0.001)$. This means that for both PVA concentra- 

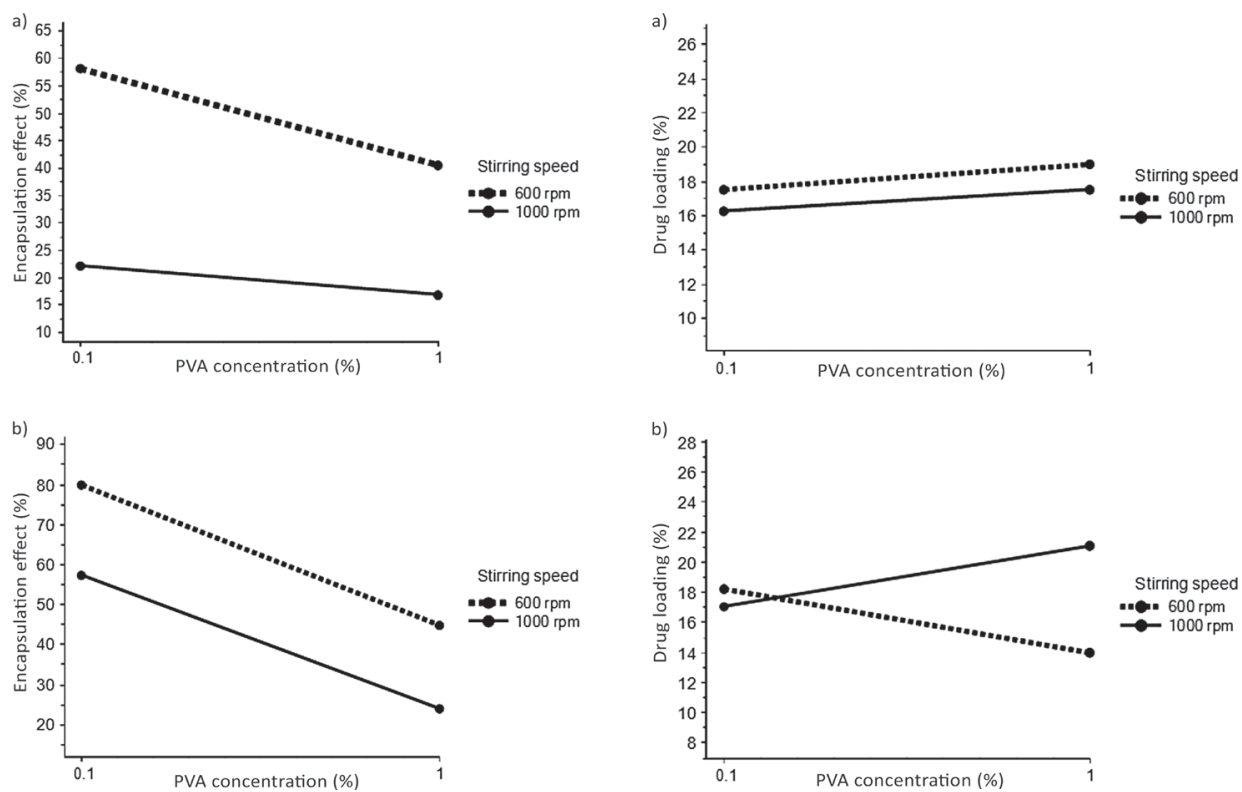

Fig. 4. Interaction plots; effect of PVA concentration on encapsulation efficiency, at various stirring speeds, for: a) ethyl acetate, b) dichloromethane.

Fig. 5. Interaction plots; effect of PVA concentration on drug loading, at various stirring speeds, for: a) ethyl acetate, b) dichloromethane.

tions an increase in stirring speed led to an equivalent decrease in drug loading. Maximum drug loading was achieved with a high PVA concentration and low stirring speed. In dichloromethane samples (Fig. 5b), the effect of PVA concentration-stirring speed interaction is apparent $(p<0.001)$. At lower PVA concentration, the influence of the stirring speed on drug loading is relatively low compared to higher PVA concentration, where an increase in the stirring speed greatly increases drug loading. However, drug loading does not reflect the loss rate of the encapsulation process and therefore it serves as a complementary characteristic to encapsulation efficiency.

\section{Drug-release behavior of microparticles}

Figs. 6 and 7 show the drug dissolution profiles performed at $\mathrm{pH}$ 6.8. The obtained release profiles were characterized as predominantly bi-phasic profiles with apparent burst release, followed by sustained drug release for at least 72 hours. The basic release pattern of PLGA microparticles is a tri-phasic profile. However, factors such as pore formation and/or closure, drug solubility, drug amount, and polymer molecular mass and/or its density can transform a tri-phasic profile into a bi-phasic one (24-26).

Burst effect. - MLR models for burst effect have good coefficients of determination, but predictive characteristic limits are reached due to greater variability between replicates. $\left(R^{2}\right.$ $>0.75, R^{2}$ predicted $<0.5$, C. V. $<18 \%$, model $p$-values: $0.05<p<0.1$, i.e., possibly significant). Despite this fact, model plots provide sufficient representation of average factor effects and 




Fig. 6. In vitro dissolution profiles of ibuprofen from PLGA samples prepared with dichloromethane as organic solvent.

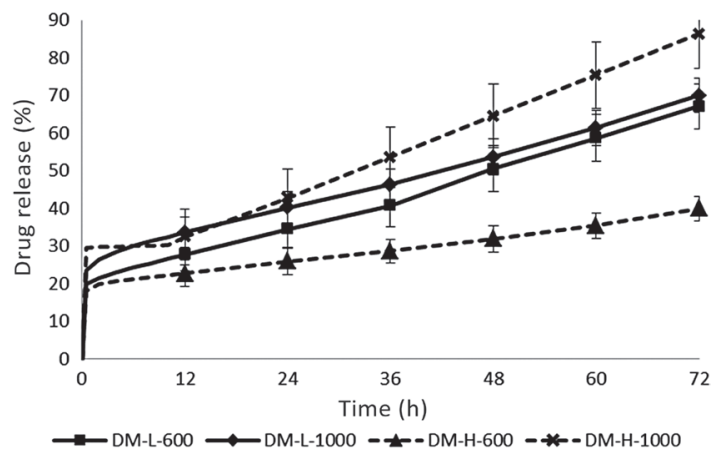

Fig. 7. In vitro dissolution profiles of ibuprofen from PLGA samples prepared with ethyl acetate as organic solvent.

models are used for approximate evaluation of the factors' influence on the effects. In samples prepared with ethyl acetate, there was possibly a significant effect $(0.05<p<0.1)$ of PVA concentration-stirring speed. At higher PVA concentration, the burst effect values were roughly the same for both stirring speeds used, whereas there was a remarkable difference at lower PVA concentration (Fig. 8a). Results of dichloromethane samples showed a significant influence of individual variable stirring speed $(p<0.05)$ on the burst effect (Fig. 8b). At $1000 \mathrm{rpm}$, the burst effect was at least six units greater than with samples prepared at 600 rpm. At $1000 \mathrm{rpm}$, smaller microparticles were prepared and therefore these samples had a larger surface area per mass unit than samples prepared at $600 \mathrm{rpm}$. This effect resulted in a higher burst effect followed by faster drug release (22). The burst effect is generally considered to be undesirable, especially because of local or systemic toxicity and shortening of release profiles (27). However, in some cases, the burst effect can be advantageous, primarily in wound treatment, targeted delivery or controlled release.

Sustained release. - Similar factor $f_{2}$ analysis also revealed the dependence of drug release on the stirring speed in dichloromethane samples prepared with $1 \%$ PVA concentra- 
Fig. 8. Interaction plots; effect of PVA concentration on burst effect, at various stirring speeds, for: a) ethyl acetate, b) dichloromethane.
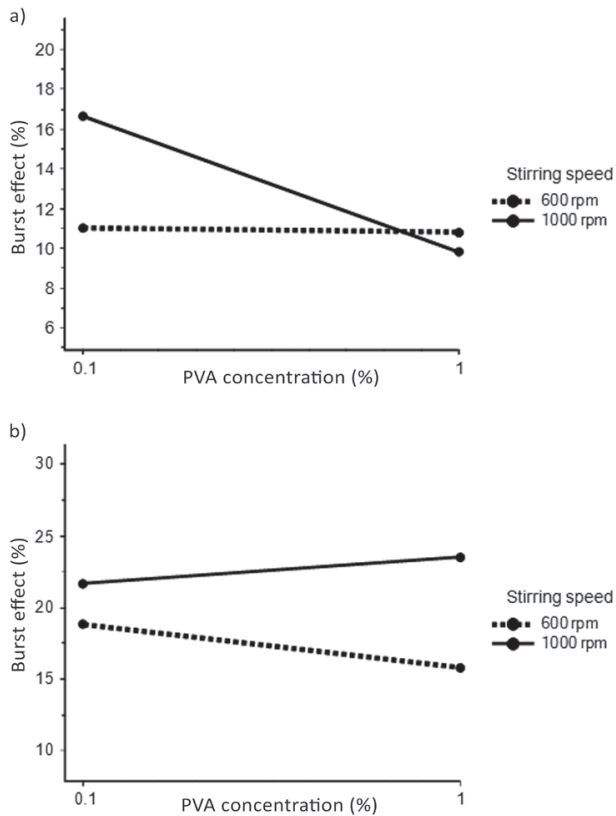

tion (DM-H-600 $\times$ DM-H-1000 $\left.f_{2}=29.9\right)$ and in ethyl acetate samples prepared with $0.1 \%$ PVA (ET-L-600 x ET-L-1000 $f_{2}=46.1$ ). Ethyl acetate samples prepared with $1 \%$ PVA and dichloromethane samples prepared with $0.1 \%$ PVA were considered to be similar (ET-H$600 \times$ ET-H-1000 $f_{2}=64.3$; DM-L-600 $\times$ DM-L-1000 $f_{2}=66.3$ ) and therefore the stirring speed alone probably had no significant influence on drug release.

PVA concentration had a noticeable impact on drug release, as shown in Figs. 6 and 7. All the samples prepared with $0.1 \%$ PVA concentration exhibited faster drug release than their equivalent counterparts prepared with $1 \%$ PVA concentration (with the exception of DM-H-1000, which showed the fastest release among all samples). For example, DM-L-600 released $67.1 \%$ of IBU within 72 hours in comparison with DM-H-600, which released 40.0 $\%$ of the drug. This is surprising, as higher PVA concentration helps produce smaller microparticles with a larger surface, just like the surfactant effect of the remaining PVA on the surface (22), and so faster drug release is to be expected. The explanation of this phenomenon could be the more even distribution of the drug inside the matrix. Higher viscosity of the $1 \%$ PVA solution can significantly lower the concentration gradient of the drug inside the particles (17). The significance of this effect was evaluated by the similarity factor $f_{2}$ analysis. Similarity factors $f_{2}$ between samples prepared with 0.1 and $1 \%$ PVA were determined. For ethyl acetate samples, the similarity factor $f_{2}$ values were 30.2 (ET-L-600 $\mathrm{x}$ ET-H-600) and 25.7 (ET-L-1000 x ET-H-1000), which indicated that there was a significant dependence of the drug amount released on the PVA concentration. However, the dichloromethane samples' similarity factor $f_{2}$ had values of 42.6 (DM-L-600 x DM-H-600) and 53.7 (DM-L-1000 x DM-H-1000). This means that in dichloromethane samples, the dependence of drug release on emulsifier concentration disappears with the increase of rotation speed, confirming the multifactorial complexity of the process (24). 


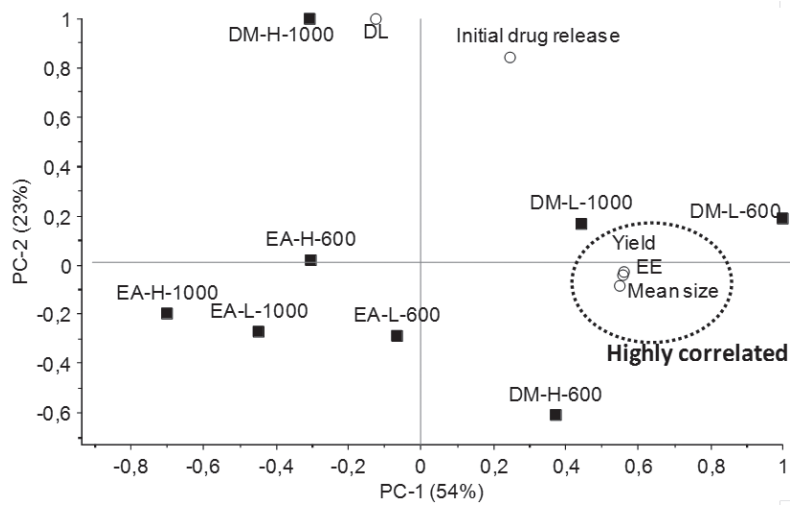

Fig. 9. PCA bi-plot displays a projection of objects (boxes) and response variables (circles) in the area of the first two components. Objects describe the performed experiment in the form: solvent (DMdichloromethane; EA-ethyl acetate)-PVA concentration (L-low; H-high)-stirring speed (600; 1000). The first two components describe $77 \%$ of total variability.

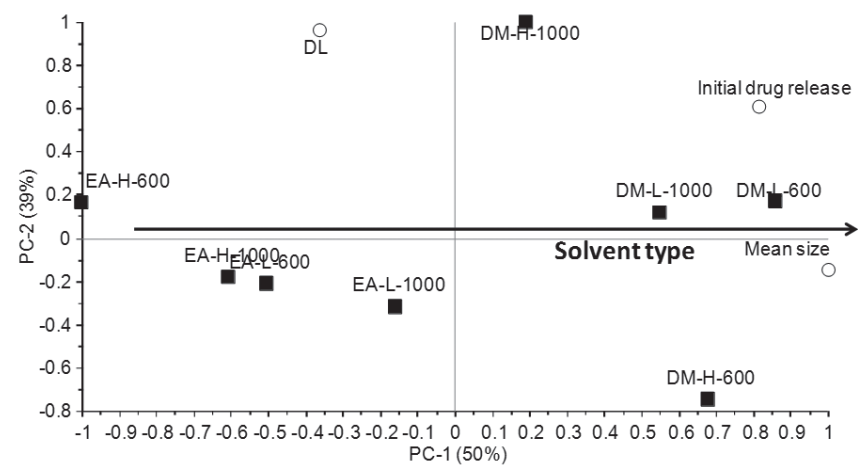

Fig. 10. PCA bi-plot displays a projection of objects (boxes) and response variables (circles) in the area of the first two components. Highly correlated variables from previous model (Fig. 9) were removed in order to take into account the remaining variability. Objects describe the performed experiment in the form: solvent (DM-dichloromethane; EA-ethyl acetate)-PVA concentration (L-low; H-high)stirring speed (600; 1000). The first two components describe $89 \%$ of total variability.

Figs. 6 and 7 show drug release differences between ethyl acetate and dichloromethane samples. The ethyl acetate samples prepared with $0.1 \%$ PVA concentration were characterized by faster drug release than samples prepared with dichloromethane. This could be explained by the different distribution of IBU in the matrix of microparticles. In the DM samples with a perfectly spherical shape, the drug could be situated more in the peripheral matrix parts, resulting in higher burst effect. On the other hand, EA samples were smaller, deformed and collapsed, could have the drug spread out more evenly around the matrix. Although the behavior of samples was different, the similarity factor $f_{2}$ between 
the ethyl acetate and the dichloromethane samples did not confirm the significance of this variable (ET-L-600 $\times$ DM-L- $600 f_{2}=54.1$; ET-L- $1000 \times$ DM-L- $1000 f_{2}=57.9$ ). Faster release from the ethyl acetate samples negated the difference caused by lower burst effect.

However, at a $1 \%$ PVA concentration, the subsequent drug release rate was approximately the same for both ethyl acetate and dichloromethane samples. This means that the curves remained parallel with a relatively wide gap. Similarity factor $f_{2}$ analysis calculated between the EA and DM samples prepared with $1 \%$ PVA revealed the significance of the solvent choice effect on dissolution profiles (ET-H-600 $\times$ DM-H-600 $f_{2}=43.0$; ET-H-1000 $\times$ DM-H-1000 $\left.f_{2}=22.7\right)$. Most likely, the higher PVA viscosity caused more evident differences in the dissolution profiles.

This interesting information was also obtained from the results of PCA analysis (Fig. 9). From the position of the symbols representing response variables, it is apparent that there was a correlation among variables-encapsulation efficiency, yield and mean microparticle size. The practical benefit of this finding could be seen in approximate prediction of these variables based on the knowledge of only one of them.

Another PCA model was designed for the study of variability among variables. Highly correlating variable encapsulation efficiency and yield were removed to prevent the overvaluation effect on the PC-1 component. This enabled a description of the mean size and initial drug release correlation (Fig. 10) and their influence on PC-1, at which the solvent type variability lies. A strong influence of organic solvent on the mentioned variables was manifested. Dichloromethane gave double values compared to ethyl acetate.

\section{CONCLUSIONS}

PLGA microparticle samples were successfully prepared by the solvent evaporation method. The full factorial design method and partial least squares PLS-2 regression were used to evaluate the impact of several formulations and process parameters used during the process. It was found that the formulation and process parameters influenced the microparticle characteristics as individual variables but also in interactions with other parameters. Multivariate data analysis was shown to be an applicable tool for identification of such relations. The results confirm the complexity of the process using the solvent evaporation method.

\section{REFERENCES}

1. D. Klose, F. Siepmann, J. F. Willart, M. Descamps and J. Siepmann, Drug release from PLGA-based microparticles: Effects of the "microparticle:bulk fluid" ratio, Int. J. Pharm. 383 (2010) 123-131; DOI: 10.1016/j.ijpharm.2009.09.012.

2. C. Regnier-Delplace, O. Thillaye Du Boullay, F. Siepmann, B. Martin-Vaca, N. Degrave, P. Demonchaux, O. Jentzer, D. Bourissou and J. Siepmann, PLGA microparticles with zero-order release of the labile anti-Parkinson drug apomorphine, Int. J. Pharm. 443 (2013) 68-79; DOI: 10.1016/j. ijpharm.2013.01.008.

3. C. Wischke and S. P. Schwendeman, Principles of encapsulating hydrophobic drugs in PLA/PLGA microparticles, Int. J. Pharm. 364 (2008) 298-327; DOI: 10.1016/j.ijpharm.2008.04.042. 
J. Vysloužil et al.: Influence of different formulations and process parameters during the preparation of drug-loaded PLGA microspheres evaluated by multivariate data analysis, Acta Pharm. 64 (2014) 403-417.

4. D. Klose, C. Delplace and J. Siepmann, Unintended potential impact of perfect sink conditions on PLGA degradation in microparticles, Int. J. Pharm. 404 (2011) 75-82; DOI: 10.1016/j.ijpharm.2010.10.054.

5. D. Klose, F. Siepmann, K. Elkharraz, S. Krenzlin and J. Siepmann, How porosity and size affect the drug release mechanisms from PLGA-based microparticles, Int. J. Pharm. 314 (2006) 198-206; DOI: 10.1016/j.ijpharm.2005.07.031.

6. S. Freitas, H. P. Merkle and B. Gande, Microencapsulation by solvent extraction/evaporation: reviewing the state of the art of microsphere preparation process technology, J. Control. Release 102 (2005) 313-332; DOI: 10.1016/j.jconrel.2004.10.015.

7. I. D. Rosca, F. Watari and M. Uo, Microparticle formation and its mechanism in single and double emulsion solvent evaporation, J. Control. Release 99 (2004) 271-280; DOI: 10.1016/j.jconrel.2004.07.007.

8. G. Paskaris and N. Bouropoulos, Swelling studies and in vitro release of verapamil from calcium alginate and calcium alginate-chitosan beads, Int. J. Pharm. 323 (2006) 34-42; DOI: 10.1016/j. ijpharm.2006.05.054.

9. S. B. Wang, A. Z. Chen, L. J. Weng, M. Y. Chen and X. L. Xie, Effect of drug-loading methods on drug load, encapsulation efficiency and release properties of alginate/poly-L arginine/chitosan ternary complex microcapsules, Macromol. Biosci. 4 (2004) 27-30; DOI: 10.1002/mabi.200300043.

10. M. Song, N. Li, S. Sun, L. R. Tiedt, W. Liebenberg and M. M. de Villiers, Effect of viscosity and concentration of wall former, emulsifier and pore-inducer on the properties of amoxicillin microcapsules prepared by emulsion solvent evaporation, Il. Farmaco. 60 (2005) 261-267; DOI: 10.1016/j. farmac.2004.11.009.

11. D. Vetchý, M. Vetchá, M. Rabišková, E. Gryczová and L. Bartošíková, Comparison in vitro felodipine release rate from the original versus generic product with controlled release of the drug, Medicina (Kaunas). 43 (2007) 326-331.

12. V. P. Shah, Y. Tsong, P. Sathe and J. P. Liu, In vitro dissolution profile comparison-statistics and analysis of the similarity factor, f2, Pharm. Res. 15 (1998) 889-896; DOI: 10.1023/A:1011976615750.

13. K. H. Esbensen, D. Guyot, F. Westad and L. P. Houmoller: Multivariate data analysis: in practice: an introduction to multivariate data analysis and experimental design, CAMO AS Publications, Oslo 2004, pp. 598.

14. P. Sansdrap and A. J. Moes, Influence of manufacturing parameters on the size characteristics and the release profiles of nifedipine from poly (DL-lactide-co-glycolide) microspheres, Int. J. Pharm. 98 (1993) 157-164; DOI: 10.1016/0378-5173(93)90052-H.

15. M. Hamoudeh and F. Hatem, Preparation, characterization and surface study of poly-epsilon caprolactone magnetic microparticles, J. Colloid. Interf. Sci. 300 (2006) 584-590; DOI: 10.1016/j. jcis.2006.04.024.

16. S. Tamilvanan, Effect of production variables on the physical characteristics of ibuprofen-loaded polystyrene microparticles, J. Microencapsul. 16 1999 411-418; DOI: 10.1080/026520499288870.

17. Y. Y. Yang, T. Chung and N. P. Ng, Morphology, drug distribution, and in vitro release profiles of biodegradable polymeric microspheres containing protein fabricated by double-emulsion solvent extraction/evaporation method, Biomaterials 22 (2001) 231-241; DOI: 10.1016/S0142-9612(00)00178-2.

18. R. M. Mainardes and R. C. Evangelista, PLGA nanoparticles containing praziquantel: effect of formulation variables on size distribution, Int. J. Pharm. 290 (2005) 137-144; DOI: 10.1016/j. ijpharm.2004.11.027.

19. T. Freytag, A. Dashevsky, L. Tillman, G. E. Hardee and R. Bodmeier, Improvement of the encapsulation efficiency of oligonucleotide-containing biodegradable microspheres, J. Control. Release 69 (2000) 197-207; DOI: 10.1016/S0168-3659(00)00299-6.

20. P. B. O'Donnell and J. W. McGinity, Preparation of microspheres by the solvent evaporation technique, Adv. Drug Deliv. Rev. 28 (1997) 25-42; DOI: 10.1016/S0169-409X(97)00049-5. 
J. Vysloužil et al.: Influence of different formulations and process parameters during the preparation of drug-loaded PLGA microspheres evaluated by multivariate data analysis, Acta Pharm. 64 (2014) 403-417.

21. Y. Capan, B. H. Woo, S. Gebrekidan, S. Ahmed and P. P. DeLuca, Influence of formulation parameters on the characteristics of poly(D, L-lactide-co-glycolide) microspheres containing poly(L-lysine) complexed plasmid DNA, J. Control. Release 60 (1999) 279-286; DOI: 10.1016/S01683659(99)00076-0.

22. S. Mao, Y. Shi, L. Li, J. Xu, A. Schaper and T. Kissel, Effects of process and formulation parameters on characteristics and internal morphology of poly(d,l-lactide-co-glycolide) microspheres formed by the solvent evaporation method, Eur. J. Pharm. Biopharm. 68 (2008) 214-223; DOI: 10.1016/j. ejpb.2007.06.008.

23. M. Li, O. Rouaud and D. Poncelet, Microencapsulation by solvent evaporation: State of the art for process engineering approaches, Int. J. Pharm. 363 (2008) 26-39; DOI: 10.1016/j.ijpharm.2008.07.018.

24. S. Fredenberg, M. Wahlgren, M. Reslow and A. Axelsson, The mechanisms of drug release in poly(lactic-co-glycolic acid)-based drug delivery systems - A review, Int. J. Pharm. 415 (2011) 34-52; DOI: 10.1016/j.ijpharm.2011.05.049.

25. N. S. Berchane, K. H. Carson, A. C. Rice-Ficht and M. J. Andrews, Effect of mean diameter and polydispersity of PLG microspheres on drug release: Experiment and theory, Int. J. Pharm. 337 (2007) 118-126; DOI: 10.1016/j.ijpharm.2006.12.037.

26. C. Berkland, K. Kim and D. W. Pack, PLG Microsphere size controls drug release rate through several competing factors, Pharmaceut. Res. 20 (2003) 1055-1062; DOI: 10.1023/A:1024466407849.

27. X. Huang and C. S. Brazel, On the importance and mechanisms of burst release in matrix-controlled drug delivery systems, J. Control. Release 73 (2001) 121-136. 